\title{
Dopamine Gates Action Potential Backpropagation in Midbrain Dopaminergic Neurons
}

\author{
Luc J. Gentet and Stephen R. Williams \\ Medical Research Council Laboratory of Molecular Biology, Cambridge CB2 2QH, United Kingdom
}

\begin{abstract}
Dopamine is released from both axonal and somatodendritic sites of midbrain dopaminergic neurons in an action potential-dependent manner. In contrast to the majority of central neurons, the axon of dopaminergic neurons typically originates from a dendritic site, suggesting a specialized computational function. Here, we examine the initiation and spread of action potentials in dopaminergic neurons of the substantia nigra pars reticulata and reveal that the displacement of the axon to a dendritic site allows highly compartmentalized electrical signaling. In response to a train of synaptic input, action potentials initiated at axon-bearing dendritic sites formed a variable trigger for invasion to the soma and contralateral dendritic tree, with action potentials often confined to the axon-bearing dendrite. The application of dopamine increased this form of electrical compartmentalization, an effect mediated by a tonic membrane potential hyperpolarization leading to an increased availability of a class of voltage-dependent potassium channel. These data suggest that the release of dopamine from axonal and somatodendritic sites are dissociable, and that dopamine levels within the midbrain are dynamically controlled by the somatodendritic spread of action potentials.
\end{abstract}

Key words: EPSP; action potential; axon; dendrite; dopamine; Parkinson's disease

\section{Introduction}

Midbrain dopaminergic neurons have important roles in brain reward and motor systems (Wichmann and DeLong, 1996; Schultz, 2006). Dopamine is released locally in the midbrain from nonaxonal, somatodendritic sites (Bjorklund and Lindvall, 1975; Geffen et al., 1976) and acts to inhibit the action potential output of dopaminergic neurons (Aghajanian and Bunney, 1977; Ruffieux and Schultz, 1980; Lacey et al., 1987; Pucak and Grace, 1994; Tepper et al., 1997; Paladini et al., 2003; Beckstead et al., 2004). This form of autoinhibition in turn controls the release of dopamine from nigrostriatal and mesolimbo-cortical axonal sites, and so is of functional importance. The mechanism(s) underlying the somatodendritic release of dopamine are controversial (Cheramy et al., 1981). Previously however, the action potential-dependent vesicular release of dopamine from somatodendritic sites of dopaminergic neurons has been described (Beckstead et al., 2004), suggesting that action potential backpropagation plays a role in the somatodendritic release of dopamine. This mechanism does not, however, exclude the somatodendritic release of dopamine by action potential independent mechanisms (Cheramy et al., 1981) such as transporter reversal (Falkenburger et al., 2001).

In contrast to many classes of central neurons, the axon of midbrain dopaminergic neurons emanates from a dendritic site (Juraska et al., 1977; Tepper et al., 1987; Häusser et al., 1995);

Received Dec. 4, 2006; revised Jan. 4, 2007; accepted Jan. 15, 2007.

This work was supported by the Medical Research Council (UK). We are grateful to $S$. Atkinson for help with anatomical procedures.

Correspondence should be addressed to Dr. Stephen R. Williams, Neurobiology Division, Medical Research Council Laboratory of Molecular Biology, Hills Road, Cambridge CB2 2QH, UK. E-mail: srw@mrc-Imb.cam.ac.uk. DOI:10.1523/JNEUROSCI.5234-06.2007

Copyright $\odot 2007$ Society for Neuroscience $\quad$ 0270-6474/07/271892-10\$15.00/0 consequently, action potentials are generated first from dendritic sites and then spread to the soma and throughout the remaining dendritic tree (Häusser et al., 1995). In vitro, the backpropagation of action potentials has been shown to be robust, with action potentials exhibiting little voltage attenuation as they spread through the dendritic tree (Häusser et al., 1995). In vivo, however, action potential backpropagation may not be as secure. Dopaminergic neurons autonomously fire action potentials in a pacemaker pattern, the waveform of such spontaneously occurring action potentials recorded in vivo, using extracellular and intracellular techniques, is made up of a sequence of distinguishable potentials that are thought to reflect the initial firing of the axon and subsequent invasion of the soma and dendrites (Guyenet and Aghajanian, 1978; Grace and Bunney, 1984b; Tepper et al., 1987, 1997; Trent and Tepper, 1991). Interestingly, these action potential components are separable, with an increased temporal dispersion and failure of the somatodendritic component apparent during burst discharges of action potentials and in response to antidromic stimuli (Guyenet and Aghajanian, 1978; Grace and Bunney, 1984b; Tepper et al., 1987, 1997; Trent and Tepper, 1991). Because ongoing somatodendritic release of dopamine occurs in vivo (Cheramy et al., 1981; Tepper et al., 1997), we hypothesized that dopamine may control the spread of action potentials through the dendritic arbor of midbrain dopaminergic neurons. To test this notion, we investigated the actions of dopamine on the propagation of action potentials through the dendritic tree of dopaminergic neurons maintained in midbrainslices using multisite whole-cell recording techniques.

\section{Materials and Methods}

Coronal mid-brain slices $(250 \mu \mathrm{m})$ were made from Wistar rats [postnatal day 15 (P15) to P22] using standard procedures following Institutional and United Kingdom Home Office guidelines. Brain-slices were 
prepared in a solution composed of (in mM) 234 sucrose, $26 \mathrm{NaHCO}_{3}$, $2.5 \mathrm{KCl}, 1.25 \mathrm{NaH}_{2} \mathrm{PO}_{4}, 0.5 \mathrm{CaCl}_{2}, 10 \mathrm{MgCl}_{2}$, and 10 glucose, and maintained in a solution composed of (in $\mathrm{mm}$ ) $125 \mathrm{NaCl}, 25 \mathrm{NaHCO}_{3}, 3 \mathrm{KCl}$, $1.25 \mathrm{NaH}_{2} \mathrm{PO}_{4}, 1 \mathrm{CaCl}_{2}, 6 \mathrm{MgCl}_{2}$, and 25 glucose (for $1 \mathrm{~h}$ at $35^{\circ} \mathrm{C}$, then at $20-24^{\circ} \mathrm{C}$ ), until transfer to a recordings chamber perfused with a solution composed of (in mM) $125 \mathrm{NaCl}, 25 \mathrm{NaHCO}_{3}, 3 \mathrm{KCl}, 1.25 \mathrm{NaH}_{2} \mathrm{PO}_{4}$, $2 \mathrm{CaCl}_{2}, 1 \mathrm{MgCl}_{2}$, and 25 glucose, gassed with $95 \% \mathrm{O}_{2}-5 \% \mathrm{CO}_{2}$ at $33-35^{\circ} \mathrm{C}$

Triple whole-cell recordings were made with identical current-clamp amplifiers (BVC 700; Dagan, Minneapolis, MN) from neurons visualized under infrared differential interference contrast (IRDIC) microscopy (Stuart et al., 1993). Pipettes (3-10 M $\Omega$ ) were filled with the following (in mM): $135 \mathrm{~K}$-gluconate, $7 \mathrm{NaCl}, 10$ HEPES, $2 \mathrm{Na}_{2}$-ATP, $0.3 \mathrm{Na}_{2}$-GTPl, 2 $\mathrm{MgCl}_{2}$, and 0.5 EGTA, pH 7.2-7.3, KOH. Simulated EPSPs were generated as ideal current sources $\left(\tau_{\text {rise }}, 0.1 \mathrm{~ms} ; \tau_{\text {decay }}, 1 \mathrm{~ms}\right)$. The kinetics of the driving current were based on those of miniature EPSCs recorded under somatic whole-cell voltage-clamp (Axopatch 200B; Molecular Devices, Union City, CA; holding potential, $-60 \mathrm{mV}$; series resistance, $10.0 \pm 0.7$ $\mathrm{M} \Omega$, compensated by $>85 \%$ in the presence of lag values of $<10 \mu \mathrm{s}$ ) in the presence of tetrodotoxin (TTX, $0.5 \mu \mathrm{M}$ ), D-2-amino-5phosphonovaleric acid $(100 \mu \mathrm{M})$, and bicuculline methiodide $(20 \mu \mathrm{M})$ ( $\tau_{\text {decay }}, 1.1 \pm 0.2 \mathrm{~ms} ; n=4$; fastest $25 \%$ of events in each neuron). The frequency and composition of barrages of simulated EPSPs were designed to mimic the postsynaptic consequence of the convergence of five subthalamic neurons firing irregularly at a mean frequency of $20 \mathrm{~Hz}$, the physiological range and pattern of subthalamic neuronal discharge in vivo (Wichmann et al., 1994). Signals were filtered at $1-10 \mathrm{kHz}$ and acquired at $10-20 \mathrm{kHz}$ using Axograph (Molecular Devices). Data were analyzed and curve fitting performed using Axograph. Numerical values are expressed as mean \pm SEM, unless otherwise stated. Statistical significance was tested with Student's $t$ test or Wilcoxon matched-pairs test.

The voltage-dependent activation (holding potential, -80 or -110 $\mathrm{mV}$; test steps, -80 to $+50 \mathrm{mV}$, in $10 \mathrm{mV}$ increments, $1.2 \mathrm{~s}$ ) and inactivation (prepulse potential, -110 to $-60 \mathrm{mV}$ in $10 \mathrm{mV}$ increments, $1.3 \mathrm{~s}$; test step, $+50 \mathrm{mV}, 1.2 \mathrm{~s}$ ) properties of pharmacologically isolated potassium currents $\left(0.5 \mu \mathrm{M}\right.$ TTX, $100 \mu \mathrm{M} \mathrm{CdCl}_{2}$, and $\left.20 \mu \mathrm{M} \mathrm{ZD} 7288\right)$ were recorded from somatic nucleated patches (access resistance, $10.2 \pm 1.0$ $\mathrm{M} \Omega$; compensated $>85 \%$ in the presence of lag $<10 \mu \mathrm{s}$ ) or outside-out patches excised from the soma or dendrites under visual control using pipettes with similar characteristics (6-9 M $\Omega$ ). Nucleated and excised patch experiments were conducted at $33-35^{\circ} \mathrm{C}$. Whole-cell recordings of $I_{\mathrm{A} \text {-like }}$ potassium currents were made with pipettes filled with the following (in mM): $120 \mathrm{CsCl}, 15 \mathrm{~K}$-gluconate, $7 \mathrm{NaCl}, 10 \mathrm{HEPES}, 2 \mathrm{Na}_{2}$-ATP, $0.3 \mathrm{Na}_{2}$-GTP, $2 \mathrm{MgCl}_{2}$, and 0.5 EGTA, pH 7.2-7.3, $\mathrm{CsOH}$, in the presence of a modified extracellular fluid [containing the following (in $\mathrm{mM}$ ): 100 $\mathrm{NaCl}, 25 \mathrm{NaHCO}_{3}, 20$ tetraethylammonium (TEA), $13 \mathrm{KCl}, 3 \mathrm{NiCl}_{2}, 2$ $\mathrm{CaCl}_{2}, 1 \mathrm{MgCl}_{2}, 0.2 \mathrm{CdCl}_{2}, 0.02 \mathrm{ZD} 7288,0.001$ TTX, and 25 glucose]. Sodium currents were recorded using pipettes filled with the following (in mM): $135 \mathrm{CsCl}, 7 \mathrm{NaCl}, 10 \mathrm{HEPES}, 2 \mathrm{Na}_{2}$-ATP, $0.3 \mathrm{Na}_{2}$-GTP, $2 \mathrm{MgCl}_{2}$, and 0.5 EGTA, pH 7.2-7.3, $\mathrm{CsOH}$, in the presence of a modified extracellular fluid [containing the following (in mM): $100 \mathrm{NaCl}, 25 \mathrm{NaHCO}_{3}$, 20 TEA, $13 \mathrm{KCl}, 54-\mathrm{AP}, 3 \mathrm{NiCl}_{2}, 3 \mathrm{CsCl}, 2 \mathrm{CaCl}_{2}, 1 \mathrm{MgCl}_{2}, 0.2 \mathrm{CdCl}_{2}$, and 25 glucose]. All whole-cell voltage-clamp recordings were made at room temperature $\left(20-24^{\circ} \mathrm{C}\right)$.

All drugs were dissolved in the recording solution. Dopamine was prepared as a stock (10 mM, with $1 \mathrm{~mm}$ ascorbate to prevent oxidation) before each application. During experiments investigating the physiological actions of potassium channel blockers, the recording solution contained amino acid receptor antagonists (10 $\mu \mathrm{M}$ 6-cyano-7nitroquinoxaline-2,3-dione, $50 \mu \mathrm{M}$ D-2-amino-5-phosphonovaleric acid, and $20 \mu \mathrm{M}$ bicuculline methiodide). In some experiments, dopamine $(100 \mu \mathrm{M})$ was locally applied by pressure ejection (5-10 s; 10 psi; Picospritzer 3; Parker Hannifin, Cleveland, $\mathrm{OH}$ ) from a pipette (similar to those used for somatic recordings) placed close $(<50 \mu \mathrm{m})$ to the soma of the recorded neuron. In some experiments TTX $(1 \mu \mathrm{M})$ was pressure applied from a pipette placed close $(<10 \mu \mathrm{m})$ to the intersection of the axon-bearing dendrite and soma.

Recordings were made from neurons of the substantia nigra pars reticulata (SNR) that contains a high density of dopaminergic neurons
(Nakanishi et al., 1987; Häusser et al., 1995; Richards et al., 1997; Falkenburger et al., 2001). Dopaminergic neurons were distinguished on the basis of their electrophysiological properties, namely the presence of time-dependent anomalous rectification and broad, somatically recorded action potential (time-dependent rectification at $-90 \mathrm{mV}$ : dopaminergic, $0.62 \pm 0.03, n=38$; GABAergic, $0.97 \pm 0.02, n=15 ; p<0.05$; action potential half-width: dopaminergic, $0.97 \pm 0.03 \mathrm{~ms}, n=44$; GABAergic, $0.38 \pm 0.02 \mathrm{~ms}, n=15 ; p<0.05$ ) (Lacey et al., 1989; Yung et al., 1991; Häusser et al., 1995). Under IRDIC microscopy, electrophysiologically identified dopaminergic neurons had a large somatic area and a characteristically thick primary dendrite $(3.1 \pm 0.1 \mu \mathrm{m}$ at $50 \mu \mathrm{m}$ from the soma; $n=12$ ) oriented toward the pars compacta region. In some experiments, Alexa 568 (10 $\mu \mathrm{M}$; Invitrogen, Eugene, OR) was included in the pipette solution, allowing the axonal and somatodendritic morphology of neurons to be imaged and captured under fluorescent microscopy in the recording setup (Retiga EXI; QImaging, Burnaby, British Columbia, Canada) and/or reconstructed from confocal optical sections $(1 \mu \mathrm{m}$; 15-45 sections; Radiance 2000; Bio-Rad, Hercules, CA) after brain-slices were mounted in vector shield mounting reagent (Vector Laboratories, Burlingame, CA). The axon of dopaminergic neurons was identified as a nontapering thin structure that emerged from the primary dendrite at a point close to the primary dendritic division and coursed through the neuropil with a bent, often torturous trajectory. These, and only these processes terminated as near spherical structures when observed under fluorescent microscopy (56 $\left.\pm 9 \mu \mathrm{m}^{2} ; n=19\right)$ (see Fig. 1), as found for cut axons in unfixed tissue from other brain areas (Palmer and Stuart, 2006; Shu et al., 2006).

\section{Results}

Previous studies have revealed that action potentials are initiated at a dendritic site in dopaminergic neurons of the substantia nigra and backpropagate without decrement throughout the dendritic tree; providing an invariant retrograde signal of neuronal output (Häusser et al., 1995). Here, we investigate the factors that control action potential backpropagation in dopaminergic neurons of the substantia nigra pars reticulata and show that the efficacy of action potential backpropagation is determined by the pattern of input used to evoke action potential firing and that the spread of action potentials (APs) through the dendritic tree is functionally gated by dopamine.

\section{Dendritic action potential initiation}

To explore the site of action potential initiation and pattern of backpropagation in dopaminergic neurons of the SNR, triple whole-cell recordings were made from somatic and dendritic sites (for the identification of neurons, see Materials and Methods) $(n=31)$ (Fig. 1$)$. When action potential firing was evoked by the generation of a long positive current step (200-600 pA; 500 $\mathrm{ms}$ ) delivered from either one of the three recording sites, action potentials were first recorded from a dendritic site and then spread to the soma and into the contralateral dendritic arbor with negligible voltage decrement (Fig. $1 A, B$ ). This robust pattern of action potential backpropagation was apparent for all action potentials of a train (Fig. 1C). Combined electrophysiological and morphological examination revealed that action potentials were first recorded from a primary dendrite that gave rise to an axon originating $135 \pm 12 \mu \mathrm{m}$ from the soma (range, $69-258 \mu \mathrm{m} ; n=$ 22) (for anatomical procedures and the criteria used to identify axons, see Materials and Methods) (Fig. 1D). Furthermore, triple recordings from neurons visualized under IRDIC microscopy revealed that action potentials were invariably recorded first from the largest calibre primary dendrite, allowing an unambiguous functional and morphological identification of the axon-bearing dendrite $(\mathrm{ABD})$ in each neuron examined $[\mathrm{ABD}$ diameter, $3.1 \pm$ $0.1 \mu \mathrm{m}$; nonaxon-bearing dendrite (nABD) diameter, $2.0 \pm 0.2$ 
$\mu \mathrm{m}$, at $50 \mu \mathrm{m}$ from the soma; $n=12 ; p<$ 0.05) (Fig. 1B). Thus, when evoked by steps of positive current, action potentials are initiated at an axon-bearing dendritic site and backpropagate faithfully throughout the dendritic tree of SNR dopaminergic neurons.

\section{Determinants of action} potential backpropagation

Action potential firing and the somatodendritic release of dopamine from SNR neurons are powerfully driven by glutamatergic inputs from the subthalamic nucleus (Nakanishi et al., 1987; Iribe et al., 1999; Falkenburger et al., 2001). We therefore investigated the spread of action potentials through the dendritic tree of dopaminergic neurons when firing was evoked by a barrage of simulated EPSPs (sEPSPs) designed to mimic the postsynaptic consequence of activity within afferent pathways (see Materials and Methods). Barrages of simulated EPSPs delivered to somatic or dendritic loci led to the generation of large-amplitude action potentials recorded from ABD sites (Fig. 2). In contrast with results obtained using current steps, however, action potentials evoked by sEPSPs failed to spread faithfully throughout the dendritic tree (Fig. 2A,B). Action potentials recorded from somatic or $\mathrm{nABD}$ sites were of variable amplitude, with large amplitude spikes intermixed with small amplitude events during a train of activity (Fig. $2 A, B)$. Consequently, when pooled for all neurons tested, the amplitude distribution of action potentials recorded from $\mathrm{nABD}$ sites was bimodal ( $n=2450$ action potentials) (Fig. 2C).

Action potentials could be divided into two classes based on their spatial-temporal profiles (Fig. 2C-E). Propagated action potentials did not attenuate as they spread from axon-bearing to nonaxon-bearing dendritic sites $(66.2 \pm 3.2 \%$ of action potentials; $\mathrm{ABD}, 69.7 \pm 1.0 \mathrm{mV}$; $\mathrm{nABD}, 72.3 \pm 0.9 ; n=31$ ) (Fig. $2 C, D$ ). The waveform of propagated APs recorded from $\mathrm{nABD}$ sites was, however, complex, with spikes typically exhibiting a clear inflection in their rising phase that was readily apparent when the derivative of voltage responses was calculated (Fig. $2 E$, arrow), a pattern of activity not observed when action potentials were evoked by long steps of positive current (compare Figs. $1 \mathrm{~B}, 2 \mathrm{E}$ ). In contrast, the second group of action potentials showed dramatic voltage attenuation as spikes spread from axon-bearing to nonaxon-bearing dendritic sites $(33.8 \pm 3.2 \%$ of action potentials; ABD, $59.9 \pm 1.5 \mathrm{mV}$; nABD, $36.5 \pm 0.7 ; p \ll 0.01 ; n=31)$ (Fig. 2C,D). At somatic and nonaxon-bearing dendritic sites, these action potentials exhibited a simple monophasic rise and lacked the prominent repolarization phase of propagated action potentials evoked by current steps or sEPSPs. Consequently, the voltage derivative of this group of action potentials was characterized by a monophasic positive component that was not followed by a large negative going repolarization phase (compare
Figs. $1 B, 2 E)$. The characteristics of the voltage waveform and calculated derivative of action potentials recorded from $\mathrm{nABD}$ and somatic sites were used to assign action potentials as failed or propagated for each recording site.

Informatively, the characteristics of the initial component of the rising phase of propagated action potentials were similar to the monophase rise of small amplitude spikes, suggesting that at nonaxon-bearing dendritic sites, small amplitude APs may represent the spread of axonal action potentials that have failed to recruit somatodendritic ion channels (initial component peak derivative of propagated APs, $37.1 \pm 2.6 \mathrm{Vs}^{-1}$; peak derivative of failed APs, $\left.32.5 \pm 2.2 \mathrm{Vs}^{-1} ; p>0.05 ; n=28\right)$. To test this idea, we pharmacologically blocked sodium channels at the intersection of the axon-bearing dendrite and soma by the local application of TTX while simultaneously recording action potentials at axon-bearing and nonaxon-bearing dendritic sites (see Material and Methods) (Fig. 3A). The local pressure application of TTX did not alter the amplitude of APs recorded close to the site of generation in the axon-bearing dendrite, but decreased the amplitude of APs as they spread to $\mathrm{nABD}$ sites [control (all APs): $\mathrm{ABD}, 69.1 \pm 4.0 \mathrm{mV} ; \mathrm{nABD}, 49.8 \pm 4.2 \mathrm{mV} ; n=5$; TTX (all APs): $\mathrm{ABD}, 69.4 \pm 4.3 \mathrm{mV}$; $\mathrm{nABD}, 36.8 \pm 3.2 \mathrm{mV} ; n=5 ; p<0.002)$. Informatively, the amplitude and waveform of action potentials 
A EPSPs at axon bearing dendrite $\|$ ABBD

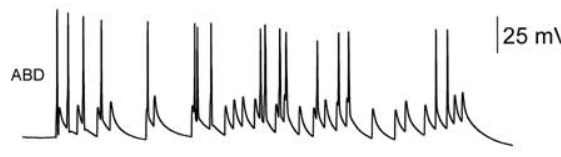

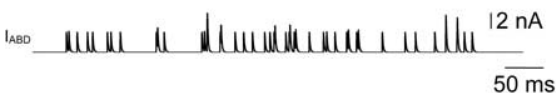

B ABBD Fropagated .

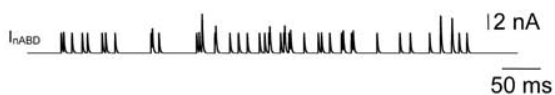

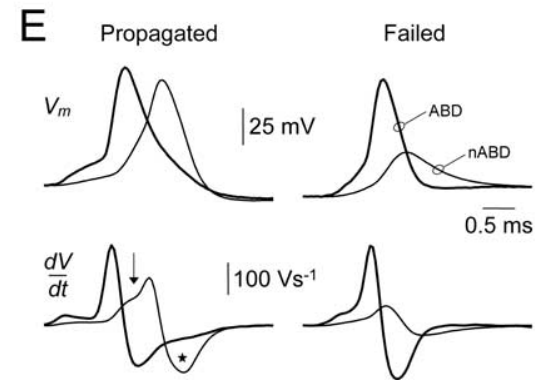

Figure 2. Synaptic activity evokes action potentials that fail to spread throughout the dendritic tree. $\boldsymbol{A}, \boldsymbol{B}$, Action potential firing evoked by a random pattern of simulated synaptic activity delivered to the axon-bearing dendrite ( $V_{A B D}$, left traces) or nonaxonbearing dendrite $\left(I_{\text {nABD }}\right)$. Note the uniform amplitude of action potentials recorded from the axon-bearing dendrite, but the variable amplitude of action potentials recorded from the nonaxon-bearing dendrite. Action potentials have been characterized as propagated (large amplitude) and failed (small amplitude) events. C, The amplitude of action potentials recorded from nonaxonbearing dendritic sites has a bimodal distribution ( $n=2450$ action potentials, $2 \mathrm{mV}$ bins). $\boldsymbol{D}$, The average amplitude of propagated and failed action potentials recorded from the nonaxon-bearing dendrite plotted as a function of distance from the axon-bearing dendritic recording site for each neuron tested. $\boldsymbol{E}$, Voltage waveform and calculated derivative of propagated and failed action potentials recorded from an axon-bearing (thick traces) and nonaxon-bearing dendrite. Note that the amplitude and waveform of propagated and failed action potentials are similar in the axon-bearing dendrite. At nonaxon-bearing dendritic sites, however, propagated action potentials are of large amplitude, whereas failed action potential are of small amplitude. Differences between the waveform of action potentials are clearly shown in the calculated derivative (bottom traces). The derivative (dV/dt) of failed action potentials show a simple monophasic waveform, whereas propagated action potentials exhibit a complex multicomponent rising phase (inflection point marked by arrow) followed by a prominent repolarization phase (asterisk).

recorded at $\mathrm{nABD}$ sites in the presence of TTX were indistinguishable from failed APs recorded under control conditions (Fig. $3 A, B$ ). Together, these data provide evidence for two general categories of action potentials, a group that propagates robustly throughout the dendritic tree of midbrain dopaminergic neurons, and a group that fail to actively spread from the axonbearing dendritic site of generation. We refer to these groups as propagated and failed action potentials, respectively.

The failure of action potentials to actively spread from the site of initiation in the axon-bearing dendrite occurred with equal preponderance whether sEPSPs were generated from $\mathrm{ABD}$ or $\mathrm{nABD}$ sites $(\mathrm{ABD}, 33.8 \pm 3.2 \%, n=31 ; \mathrm{nABD}, 31.4 \pm 3.5 \%, n=$ 8) (Fig. $2 A, B$ ). To investigate the site at which action potentials failed in dopaminergic neurons, we calculated the amplitude of failed and propagated action potentials at somatic, $A B D$, and nABD sites (Fig. $4 A$ ) after the categorization of action potentials according to their characteristic voltage waveforms. Analysis revealed that the amplitude of failed and propagated action potentials were similar at ABD sites (failed/propagated amplitude ratio, $0.85 \pm 0.01 ; n=31$ ) (Fig. $4 A$ ). In contrast, at somatic and nABD sites, the amplitude of failed action potentials were dramatically smaller than propagated action potentials (soma, $0.51 \pm 0.01$; $\mathrm{nABD}, 0.51 \pm 0.01$ ) (Fig. $4 A$ ). To define the site at which action potentials fail in dopaminergic neurons, we made dendritic recordings from sites along the axon-bearing dendrite using pipettes filled with a fluorescent dye. At the end of the experiment, we calculated the distance of the dendritic recording pipette from the site of axon origin (for criteria used to identify axons, see Materials and Methods) (Fig. 4B). When recorded close to the site of axonal origin, the amplitude of failed and propagated action potentials were similar (Fig. $4 B$, top image, red traces) (action potential failure was defined independently at the soma). In contrast, when recorded at dendritic sites distant to the origin of the axon, failed action potentials were of small amplitude (Fig. $4 B$, bottom image and traces). Surprisingly, we found that the amplitude of failed action potentials, as represented by the amplitude ratio of failed and propagated APs, declined steeply with distance from the axon origin with a space constant of just $26 \mu \mathrm{m}$ ( $n=26$; failed and propagated APs grouped according to somatic waveforms) (Fig. 4C). To further examine this behavior, we made dual dendritic recordings from the axon-bearing dendrite; in all cases action potentials were detected first at distal dendritic sites, where little disparity was found between the amplitude of failed and propagated action potentials (failed/propagated AP ratio, $0.90 \pm 0.01 ; 160 \pm 15 \mu \mathrm{m}$ from the soma; $n=8$ ) (Fig. $4 D$ ). In contrast, at proximal $\mathrm{ABD}$ sites, failed action potentials were significantly smaller than propagated action potentials (failed/propagated AP ratio, $0.71 \pm 0.03 ; 76 \pm 7 \mu \mathrm{m}$ from the soma; $p<0.01 ; n=8$ ) (Fig. $4 D$ ). When taken together, these data indicate that action potentials are reliably initiated at axonal sites, but can fail as they spread along the axon-bearing dendrite toward the soma, a finding that reveals a highly compartmentalized operation of dopaminergic neurons.

The time taken for action potentials to spread through the dendritic tree of dopaminergic neurons was highly variable (Fig. 5). Conduction time, as calculated from the peak-to-peak time difference between action potentials recorded from axon-bearing and nonaxon-bearing dendritic sites was found to be fastest and least variable for failed APs (slope of regression line, $1.6 \mathrm{~m} / \mathrm{s}$ ) (Fig. $5)$. In contrast, the conduction time of propagated APs was on average slower and showed pronounced event-to-event variability (Fig. $5 A-C$ ). This behavior resulted from the composite nature of propagated APs. Inspection of action potential voltage waveforms and calculated first derivatives clearly demonstrated the variable separation between the first and second components of the AP, with some action potentials exhibiting a slow ramplike depolarization between components (Fig. 5A, compare top, bottom traces). These data therefore demonstrate that the spread of axonal action potentials to the soma and nonaxon-bearing dendritic tree is insecure, either failing to achieve, or achieving with variable fidelity, the voltage necessary to recruit somatodendritic sodium channels. This process not only resulted in the slow 


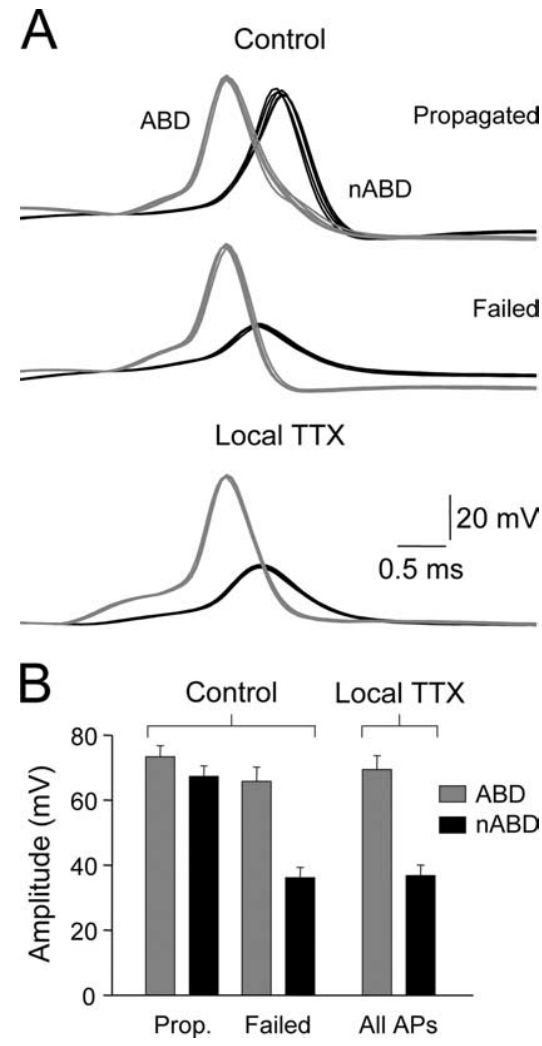

Figure 3. Failed action potentials do not recruit somatodendritic sodium channels. A, Simultaneous recording of action potentials from an axon-bearing and nonaxon-bearing dendrite. Action potentials recorded under control were divided into two classes, propagated (top overlain traces) and failed (bottom traces). The application of TTX (1 $\mu \mathrm{m})$ to the axon-bearing dendrite-soma border blocked the propagation of action potentials. $\boldsymbol{B}$, Summary data showing the amplitude of action potentials recorded under control and TTX. In the presence of TTX, the amplitude of action potentials recorded from nonaxonal bearing dendritic sites are similar to those of failed action potentials under control ( $n=5$ neurons). Bars indicate mean \pm SEM.

and temporally imprecise spread of action potentials through the dendritic tree (Fig. $5 C$ ), but also acted to sculpt the waveform of action potentials recorded at $\mathrm{ABD}$ sites (Fig. $5 \mathrm{~A}$, bottom traces). Action potentials recorded from the ABD were often biphasic, exhibiting a second small amplitude spike-like component, a behavior most evident for events that gave rise to APs showing a wide temporal separation of components (Fig. 5A, bottom traces). Under these conditions, the somatodendritic component of the nABD AP preceded and apparently drove the second component of the $\mathrm{ABD}$ action potential (Fig. $5 \mathrm{~A}$ ). Therefore, the wide temporal separation of AP components can form a situation where regenerative events reverberate between $\mathrm{ABD}$ and $\mathrm{nABD}$ sites, a process that we refer to as action potential reflection (Fig. 5A).

\section{Dopamine gates action potential backpropagation}

Dopamine released locally within the midbrain is believed to control action potential firing of dopaminergic neurons, a form of autoinhibition (Aghajanian and Bunney, 1977; Ruffieux and Schultz, 1980; Lacey et al., 1987; Pucak and Grace, 1994; Tepper et al., 1997; Paladini et al., 2003; Beckstead et al., 2004). We therefore investigated whether exogenous application of dopamine modulated the pattern of action potential backpropagation. When action potential firing was generated in response to a barrage of sEPSPs, the application of dopamine transformed the pattern of action potential backpropagation (Fig. $6 \mathrm{~A}$ ), reshaping
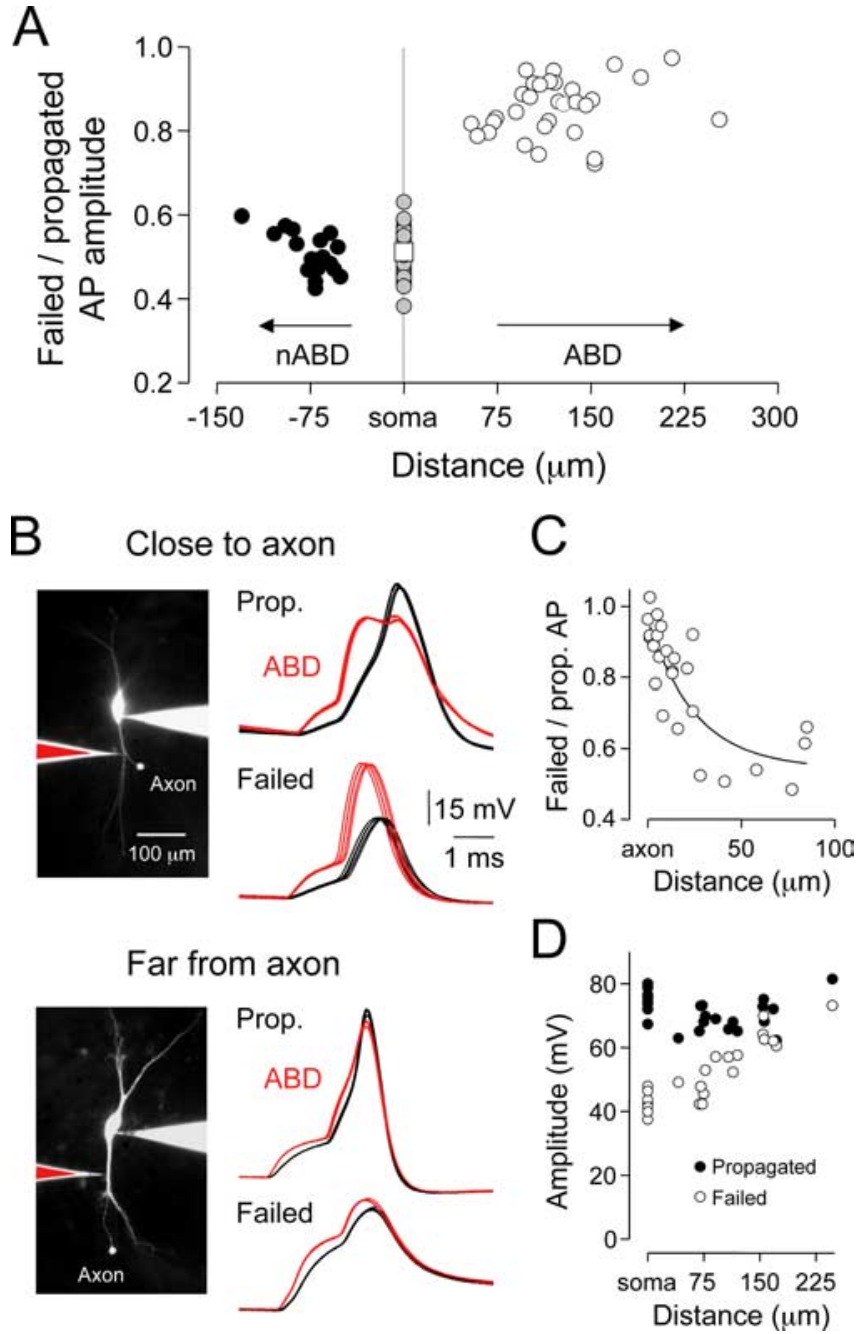

Figure 4. Site of action potential failure. $\boldsymbol{A}$, Relationship between the amplitude of failed and propagated action potentials when recorded from axon-bearing, somatic, and nonaxonbearing dendritic sites. Grouped data revealed that the amplitude of failed and propagated action potentials are similar at ABD sites but dissimilar from somatic and $\mathrm{nABD}$ sites. Action potentials were characterized by spike shape at the soma. $\boldsymbol{B}$, Action potentials fail in the axonbearing dendrite. The disparity between the amplitude of failed and propagated action potentials is negligible when action potentials are recorded close to the dendritic origin of the axon (red traces, top photomicrograph; pipette to axon separation, $6 \mu \mathrm{m}$ ) but pronounced when recorded from a location far from the site of axon origin (bottom photomicrograph; pipette to axon separation, $58 \mu \mathrm{m}$ ). Black traces represent simultaneous somatic recordings. C, Summary graph showing the amplitude ratio of failed and propagated action potentials as a function of recording distance from the axon origin. Note the large amplitude disparity that evolves with a space constant of $26 \mu \mathrm{m}$ (line). $\boldsymbol{D}$, Amplitude of failed (open symbols) and propagated action potentials recorded simultaneously from distal axon-bearing dendritic, proximal axon-bearing dendritic, and somatic sites. Note the uniform amplitude of propagated action potentials, but the steep distance-dependent decrement of the amplitude of failed action potentials.

the bimodal amplitude distribution of action potentials recorded from somatic and nABD sites (control median, $65.5 \mathrm{mV}, n=$ 2355 APs; dopamine median, $45.6 \mathrm{mV}, n=1902 \mathrm{APs}$ ) (Fig. 6B). When the median values for action potential distributions for individual neurons were pooled, we found that dopamine significantly decreased the amplitude of action potentials recorded from somatic or nABD sites (control, $60.7 \pm 3.4 \mathrm{mV}$; dopamine $(100 \mu \mathrm{M}$ ), $53.2 \pm 3.6 \mathrm{mV} ; p<0.01 ; n=23$ ) (Fig. 6C). However, dopamine did not alter the amplitude of action potentials recorded from ABD sites (control, $62.8 \pm 1.5 \mathrm{mV}$; dopamine (100 $\mu \mathrm{M}$ ), $63.2 \pm 1.6 \mathrm{mV} ; n=23$ ) (Fig. $6 \mathrm{C}$ ). Notably, the decrease of 
A Propagated APs

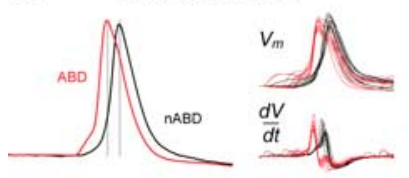

Failed APs

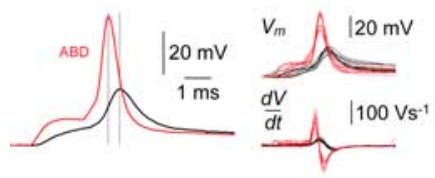

Reflected APs
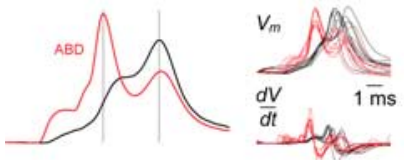

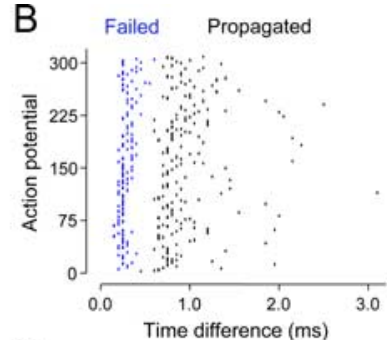

C

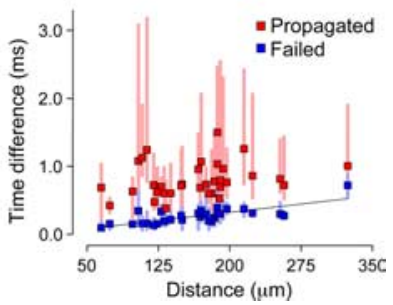

Figure 5. Infidelity of action potential backpropagation. $\boldsymbol{A}$, Representative voltage records show the wide trial-to-trial temporal separation between action potentials recorded from an axon-bearing (red) and nonaxon-bearing dendritic site. Action potentials were divided into three classes based on the characteristic of their voltage waveform and temporal separation. The inset voltage traces and calculated derivative $(\mathrm{dV} / \mathrm{dt})$ show the variability for each class of event. $\boldsymbol{B}$, Raster plot showing the variable time difference between action potentials recorded from an axon-bearing and non axon-bearing site; each line represents an action potential. Note the relatively fast spread and narrow temporal distribution of failed action potentials (blue). $\boldsymbol{C}$, Pooled data showing the time taken for propagated (red) and failed action potentials to spread from an axon-bearing dendritic site to a nonaxon-bearing dendrite. For each neuron, average values were calculated from raster plots as shown in $\boldsymbol{B}$, where vertical lines represent the maximum and minimum time taken for the spread of action potentials between recording loci. The line through failed action potential points represents a linear regression and has a slope of $1.6 \mathrm{~m} / \mathrm{s}$.

AP amplitude at nonaxon-bearing sites was accompanied by a significant increase in the event-to-event variation of action potential amplitude, suggesting that dopamine led to an alteration of the proportion of propagated and failed APs [control coefficient of variation $(\mathrm{CV}), 0.29 \pm 0.02$; dopamine $(100 \mu \mathrm{M}) \mathrm{CV}$, $0.33 \pm 0.02$; Wilcoxon matched-pairs test, $p<0.01 ; n=23$ ). Indeed, when action potentials were sorted according to their characteristic voltage waveforms, dopamine was found to dramatically increase the occurrence of failed APs (control, $31.9 \pm$ $3.8 \%$; dopamine $(100 \mu \mathrm{M}), 47.9 \pm 3.5 \% ; n=23$; Wilcoxon matched-pairs test, $p<0.001$ ) (Fig. 6 A). Importantly, the mean amplitude of both propagated and failed action potentials was not altered by dopamine in each neuron tested (Fig. 6D), suggesting that dopamine gates the spread of action potentials by controlling the fraction of spikes that fail to actively spread from the axon-bearing dendrite. The control of action potential propagation by dopamine was accompanied by a hyperpolarization of the membrane potential (3.2 $\pm 0.2 \mathrm{mV} ; n=35)$ and was blocked by the $\mathrm{D}_{2}$ receptor antagonist sulpiride (100 nm; $\left.n=7\right)$. Next, we explored how the activation of $\mathrm{D}_{2}$ receptors influenced both pacemaker and synaptically evoked patterns of action potential firing by the delivery of barrages of sEPSPs during periods of pacemaker action potential firing. The activation of $\mathrm{D}_{2}$ receptors by quinpirole $(100 \mu \mathrm{M})$ decreased the frequency of pacemaker AP firing (control, $1.52 \pm 0.38 \mathrm{~Hz}$; quinpirole, $0.38 \pm 0.13 \mathrm{~Hz} ; p<$ $0.001 ; n=19$ ) and increased the proportion of failed action potentials at nonaxon-bearing dendritic sites evoked during sEPSP barrages (control, $21.8 \pm 4.1 \%$; quinpirole, $35.0 \pm 4.3 \%$; Wilcoxon matched-pairs test, $p<0.001 ; n=19$ ), with effects accompanied by a tonic membrane potential hyperpolarization (control, $-44.9 \pm 0.9 \mathrm{mV}$; quinpirole, $-50.7 \pm 1.3 \mathrm{mV}$ ). Dopamine receptor activation therefore controls both the action potential output and the fidelity of backpropagation through the dendritic tree of dopaminergic neurons.

\section{Function and distribution of $I_{\mathrm{A}-\mathrm{like}}$ channels}

The backpropagation of action potentials in cortical pyramidal neurons is controlled by the availability of dendritic sodium and potassium channels, in particular $I_{\mathrm{A}-\text { like }}$ channels (Hoffman et al., 1997; Williams and Stuart, 2000b; Stuart and Häusser, 2001; Bernard and Johnston, 2003; Bernard et al., 2004). Dopaminergic neurons exhibit a prominent whole-cell $I_{\mathrm{A} \text {-like }}$ potassium current that has been suggested to control the rate of action potential firing (Liss et al., 2001; Yang et al., 2001). We therefore investigated the possibility that $I_{\mathrm{A} \text {-like }}$ potassium channels control action potential backpropagation in dopaminergic neurons. First, we explored the properties of potassium channels in nucleated patches excised from dopaminergic neurons. Pharmacologically isolated potassium currents were characterized by a prominent transient current (peak amplitude $1.59 \pm 0.16 \mathrm{nA}$ ) that declined to a steady state $(0.16 \pm 0.02 \mathrm{nA})$ biexponentially $\left(\tau_{\text {fast }}, 21.6 \pm 1.9\right.$ ms, 67. 3\%; $\tau_{\text {slow }}, 690.1 \pm 59.6 \mathrm{~ms}, 32.7 \% ; n=6$ ). Ensemble potassium currents could be pharmacologically dissected into two components, a fast component that was sensitive to 4 -aminopyridine [ 4 -AP ( $5 \mathrm{~mm}$ ), $86.7 \pm 2.3 \%$ reduction of peak current; $n=6$ ], and a sustained component sensitive to TEA (20 $\mathrm{mm}, 79.9 \pm 3.1 \%$ reduction of steady-state current; $n=6$ ), The properties of the transient component are therefore indicative of an $I_{\text {A-like }}$ potassium current.

Next, we explored the distribution and functional properties of $I_{\mathrm{A}-\mathrm{like}}$ channels by excising outside-out patches from the soma and dendrites of dopaminergic neurons, in the presence of TEA $(20 \mathrm{~mm})$ to block sustained potassium currents. $I_{\mathrm{A} \text {-like }}$ channels were found at a high density throughout the somatodendritic arbor, when activated in response to a $100 \mathrm{mV}$ test step (500 ms) delivered from an intrapipette potential of $-80 \mathrm{mV}$ (Fig. 7A). On average, however, channel density was greatest at somatic sites and declined at $\mathrm{ABD}$ and $\mathrm{nABD}$ sites (soma, $189.1 \pm 26.5 \mathrm{pA}, n=$ 57; ABD, $99.8 \pm 11.6 \mathrm{pA}, 94.3 \pm 5.6 \mu \mathrm{m}$ from soma, $n=40$; nABD, $105.8 \pm 23.3 \mathrm{pA}, 75.7 \pm 4.5 \mu \mathrm{m}$ from soma, $n=20$ ) (Fig. $7 B)$. To explore the functional properties of $I_{\mathrm{A}-\text { like }}$ channels, we examined the voltage-dependence of activation and inactivation processes. The activation properties of $I_{\mathrm{A}-\text { like }}$ ensemble channel activity were found to be similar for somatic and dendritic sites (for voltage protocols, see Materials and Methods) (Fig. 7D). To our surprise, however, we observed that the inactivation properties of $I_{\mathrm{A}-\text { like }}$ channels were determined in a region specific manner (Fig. 7C,D). At dendritic sites, $I_{\mathrm{A} \text {-like }}$ channels were close to full inactivation at an intrapipette potential of $-60 \mathrm{mV}(92.3 \pm$ $2.0 \% ; n=14)$. In contrast, in patches excised from somatic sites $I_{\mathrm{A} \text {-like }}$ channels were only $78.7 \pm 7.3 \%$ inactivated at $-60 \mathrm{mV}$ ( $n=10 ; p<0.05$ compared with dendritic sites) (Fig. $7 C, D)$, a site-dependent difference in steady state inactivation also apparent from an intrapipette potential of $-50 \mathrm{mV}$ (dendrite, $97.8 \pm$ 0.7\%, $n=14$; soma, $93.8 \pm 1.7 \%, n=10$; $p<0.05$ ) (Fig. $7 D$ ). These findings indicate that the majority of dendritic $I_{\mathrm{A}-\text { like }}$ channels are inactivated, but a significant fraction of somatic $I_{\mathrm{A} \text {-like }}$ channels are available for activation within the membrane potential $(-60$ to $-50 \mathrm{mV})$ range over which action potential are generated in dopaminergic neurons. To directly explore whether somatic $I_{\mathrm{A} \text {-like }}$ channels are recruited by action potentials, we recorded pharmacologically isolated transient potassium currents from nucleated patches in response to a somatic action potential 
shaped waveform. The integral of leaksubtracted outward currents increased in a voltage-dependent manner as action potential-shaped voltage commands were delivered from progressively negative membrane potentials $(-55 \mathrm{mV}, 24 \pm 7$, $-65 \mathrm{fAs} ;-65 \mathrm{mV}, 79 \pm 17 \mathrm{fAs} ;-75 \mathrm{mV}$, $176 \pm 25 \mathrm{fAs} ; n=6 ; n / 5$ subtraction protocol; from each membrane potential, the action potential voltage command was scaled to attain a peak voltage of $+35 \mathrm{mV}$ ). The $I_{\mathrm{A}-\text { like }}$ channel blocker 4-AP strongly inhibited action potential evoked currents, confirming that somatic $I_{\mathrm{A}-\text { like }}$ channels are recruited by action potentials across a physiological relevant membrane potential range [percentage reduction in 4-AP (2 mM), -55 mV, $78 \pm 11 \%$; 65 $\mathrm{mV}, 71 \pm 6 ;-75 \mathrm{mV}, 81 \pm 3.9 ; n=6$ ) (data not shown). Consequently, we investigated whether $I_{\mathrm{A}-\text { like }}$ channels influence the backpropagation of action potentials. Blockade of $I_{\mathrm{A}-\text { like }}$ channels with 4-AP ( 3 or $5 \mathrm{~mm}$; $n=14$ ) prevented the failure of action potential backpropagation in all neurons examined (control, $34.5 \pm 6.6 \%$ ) (Fig. $8 A$ ). This dramatic increase in the security of action potential backpropagation was accompanied by a significant increase in the amplitude of action potentials recorded from both $\mathrm{ABD}$ and $\mathrm{nABD}$ sites when the median values of AP amplitude were pooled across neurons (Fig. $8 B, C$ ). This effect did not solely result from an alteration of the proportion of failed and propagated action potentials, as the mean amplitude and duration of propagated action potentials recorded from nABD sites was controlled by 4-AP sensitive potassium channels (control, $72.9 \pm 1.3 \mathrm{mV}, 1.1 \pm 0.1 \mathrm{~ms}$; 4-AP (3-5 mM), $78.1 \pm 2.1 \mathrm{mV}, 1.7 \pm 0.1 \mathrm{~ms} ; p<0.05$ ) (Fig. $8 C$ ). Together, these data show that 4 -AP-sensitive potassium channels play a critical role in the control of action potential backpropagation in dopaminergic neurons.

\section{How does dopamine gate AP backpropagation?}

The activation of G-protein-coupled receptors has been shown to modulate the function of voltage-activated ion channels (Carr et al., 2003). To examine the mechanisms underlying the gating of action potential backpropagation by dopamine, we first explored whether the activation of $\mathrm{D}_{2}$ receptors directly modulates the function of sodium and $I_{\mathrm{A} \text {-like }}$ potassium channels. Under wholecell voltage-clamp, $\mathrm{D}_{2}$ receptor activation did not modulate the properties of pharmacologically isolated sodium currents, leaving the availability of sodium channels unchanged when evoked by positive voltage pulses (to $-10 \mathrm{mV}$ ) from a potential of -60 or $-100 \mathrm{mV}\left[V_{\mathrm{H}}-60 \mathrm{mV}\right.$ : control, $2.76 \pm 0.28 \mathrm{nA}$; quinpirole (100 $\mu \mathrm{M}), 2.71 \pm 0.28 \mathrm{nA} ; V_{\mathrm{H}}-100 \mathrm{mV}$ : control, $3.61 \pm 0.40 \mathrm{nA}$; quinpirole, $3.66 \pm 0.40 \mathrm{nA} ; n=11]$. Similarly, $\mathrm{D}_{2}$ receptor activation did not influence the amplitude or the voltage-dependent properties of pharmacologically isolated whole-cell $I_{\mathrm{A} \text {-like }}$ currents (peak current: control, $12.54 \pm 0.58 \mathrm{nA}$; quinpirole (100 $\mu \mathrm{M}), 12.70 \pm 0.57 \mathrm{nA}$; activation $V_{\text {half }}$ : control, $8.5 \mathrm{mV}$; quinpirole, $8.8 \mathrm{mV}$; inactivation $V_{\text {half: }}$ control, $-63.0 \mathrm{mV}$; quinpirole,
$-64.0 \mathrm{mV} ; n=11$ ) (for details of voltage protocols, see Materials and Methods). These data indicate that dopamine does not directly modulate the function of sodium and potassium channels, but may act by altering the availability of these channels because of the membrane hyperpolarization generated by $\mathrm{D}_{2}$ receptor activation. To test this idea, we examined the proportion of failed action potentials under control conditions and after direct membrane hyperpolarization, generated by the passage of negative current through the somatic recording electrode. Membrane potential hyperpolarization quantitatively replicated the action of dopamine, increasing the proportion of failed APs (control, $38.9 \pm 5.6 \%$; hyperpolarization, $51.4 \pm 5.3 \%$; control, $-60.1 \pm$ $0.4 \mathrm{mV}$; hyperpolarization, $-62.6 \pm 0.6 \mathrm{mV} ; n=13$ ). Moreover, if the membrane potential hyperpolarization generated by dopamine was compensated for by somatic injection of positive current, the actions of dopamine on the failure rate of action potentials were largely mitigated (control, $33.7 \pm 4.6 \%$; dopamine plus depolarization, $37.6 \pm 3.2 \% ; n=11)$. These data indicate that the spread of action potentials in dopaminergic neurons is tightly controlled by the voltage-dependent availability of sodium and potassium channels. To test how the actions of dopamine are influenced by the availability of $I_{\mathrm{A} \text {-like }}$ potassium channels we explored if the actions of dopamine were occluded by the pharmacological blockade of $I_{\mathrm{A}-l i k e}$ channels. In all neurons examined, the dopamine mediated increase in action potential failure was abolished by the previous blockade of $I_{\mathrm{A} \text {-like }}$ channels $(n=9)$ (Fig. 8A), Consequently, in the presence of 4-AP, dopamine 

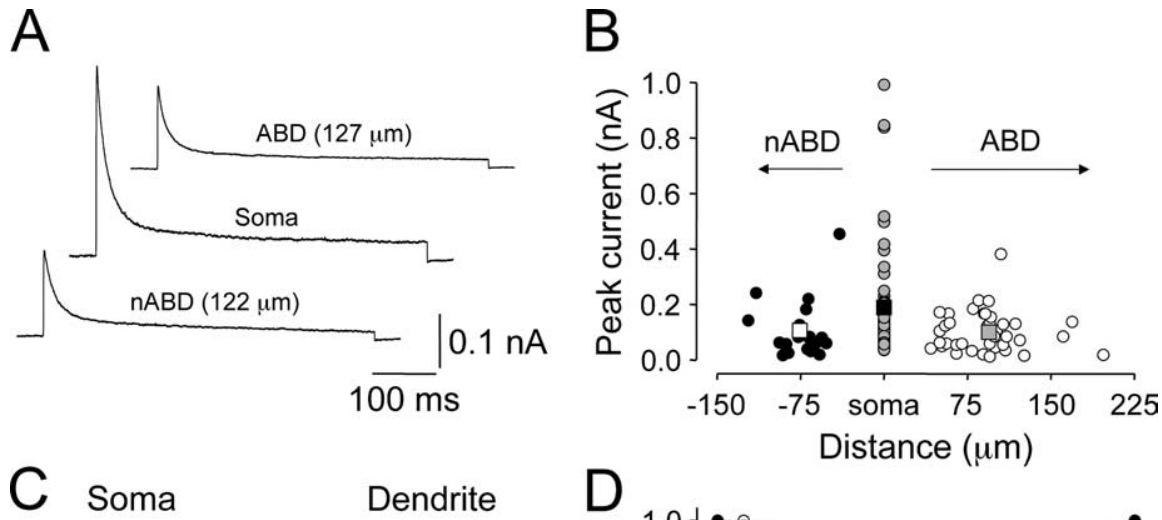

\section{Dendrite}
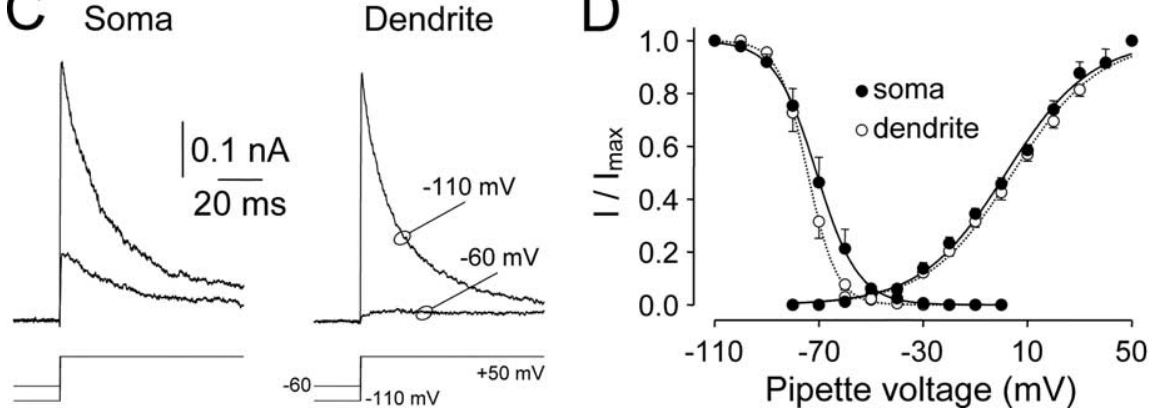

Figure 7. Distribution and properties of $I_{\text {A-like }}$ potassium channels. $\boldsymbol{A}$, Representative traces of ensemble potassium channe activity in outside-out patches excised from the soma, $A B D$, and $n A B D$ at the indicated distances form the center of the soma. Channel activity was evoked by the delivery of a positive voltage step $(100 \mathrm{mV})$ from a holding potential of $-80 \mathrm{mV}$. B, Somatodendritic distribution of $I_{\text {A-like }}$ potassium channel density. Square symbols represent the average amplitude of ensemble $I_{\text {A-like }}$ potassium channel activity at the indicated sites. Note the twofold greater density of somatic channels. $C$, The voltage-dependent inactivation properties of $I_{\text {A-like }}$ potassium channels are site-dependent. Traces show the influence of a hyperpolarizing prepulse (to -60 or $-110 \mathrm{mV}, 1.3 \mathrm{~s}$ ) on ensemble channel activity evoked by a positive voltage step (to $+50 \mathrm{mV}$ ) when recorded from a somatic or axon-bearing dendritic site. $\boldsymbol{D}$, Pooled data show the voltage-dependence of steady state activation and inactivation properties of $I_{\text {A-like }}$ potassium channels in patches excised from somatic $(n=10$; filled symbols) and dendritic sites $(n=14)$. Relationships are fit with single Boltzmann functions. Note the disparity between the shape of inactivation curves, which results in an increased fraction of available somatic $I_{\text {A-like }}$ potassium channels across a range $(-70$ to $-50 \mathrm{mV})$ of potentials. Symbols represent mean \pm SEM.

failed to alter the amplitude of action potentials at ABD and nABD sites (Fig. $8 D$ ). In contrast, 4-AP, did not block the dopamine induced membrane potential hyperpolarization [dopamine plus 4 -AP ( 3 or $5 \mathrm{~mm}$ ), $2.3 \pm 0.3 \mathrm{mV} ; n=9]$.

\section{Discussion}

The major finding of the present work is that the spread of action potentials through the dendritic tree of dopaminergic neurons of the substantia nigra pars reticulata is influenced by dopamine; the mechanisms underlying these results and their functional implications are discussed below.

In contrast to other classes of central neurons, the axon of midbrain dopaminergic neurons originates at a dendritic site (Juraska et al., 1977; Tepper et al., 1987; Häusser et al., 1995), an anatomical arrangement that suggests a functional isolation of the axon from the soma and the majority of the dendritic tree. A reason for the distal dendritic origin of the axon could be the electrical isolation of the majority of the neuron from action potentials (Grace, 1990; Tepper et al., 1997) that are initiated from axonal sites (Stuart et al., 1997). Action potential backpropagation from the axon-bearing dendrite of dopaminergic neurons to the soma and throughout the dendritic tree has, however, been shown to occur with great fidelity in vitro (Häusser et al., 1995). However, in vivo, a clear separation of action potential components thought to represent the firing of the axon and subsequent invasion of the soma and dendrites has been observed during ongoing pacemaker activity and action potential burst firing (Guyenet and Aghajanian, 1978; Grace and Bunney, 1984a,b; Tepper et al., 1987, 1997; Trent and Tepper, 1991). Indeed, a complex multiphase action potential waveform is considered a defining characteristic of midbrain dopaminergic neurons recorded in vivo (Guyenet and Aghajanian, 1978; Ruffieux and Schultz, 1980; Grace and Bunney, 1984a,b; Tobler et al., 2005). This disparity prompted us to re-evaluate the properties of action potential backpropagation in dopaminergic neurons in vitro. We find that the soma and majority of dendritic tree of dopaminergic neurons can be electrically isolated from axonal action potential firing when action potentials are evoked by a barrage of simulated EPSPs, generated from somatic or dendritic sites. This occurs because axonal action potentials form a variable trigger for the activation of somatodendritic sodium channels; consequently, action potentials initiated at an axon-bearing dendritic site can fail to invade the soma and nonaxonbearing dendrites. Such electrical failure of backpropagating APs (BPAPs) has biochemical consequences, as shown by the lack of slow calcium-dependent afterhyperpolarizing potentials after failed BPAPs, which are typical of full-blown somatic action potentials (Nakanishi et al., 1987; Lacey et al., 1989; Grace, 1990; Yung et al., 1991; Häusser et al., 1995). Failure of backpropagation was only apparent when action potential firing was generated in response to sEPSPs, and not reproduced when action potentials were evoked by long steps of positive current delivered at somatic or dendritic sites from the same loci and at the same membrane potential as trains of sEPSPs. Thus, the fidelity of action potential backpropagation is influenced by the trajectory of voltage deviations and not simply by the voltage achieved. These findings are based on the injection of EPSCshaped waveform with kinetics determined from the time course of miniature EPSCs. Temporal jitter in the release process may, however, slow the time course of compound PSCs (Isaacson and Walmsley, 1995; Williams et al., 1998). Because such jitter is minimal at physiological temperatures, it will not appreciably slow the time course of EPSPs (Stuart and Sakmann, 1995; Williams and Stuart, 2002).

The efficacy of action potential backpropagation in central neurons is determined by the density of voltage-activated channels and the morphology of the dendritic tree (Häusser et al., 2000; Williams and Stuart, 2000a; Vetter et al., 2001; Bernard and Johnston, 2003). In cortical pyramidal neurons, dendritic membrane potential depolarization has been found to augment the amplitude of BPAPs because of the increased recruitment of dendritic sodium channels (Williams and Stuart, 2000b; Stuart and Häusser, 2001) and the inactivation of transient $I_{\mathrm{A}-l i k e}$ potassium channels (Hoffman et al., 1997). Consequently, when action potentials are paired with a train of dendritic EPSPs in hippocampal pyramidal neurons, the amplitude of BPAPs increases through- 
out the train (Magee and Johnston, 1997; Johnston et al., 2000). In contrast, we find that failed APs are intermixed with reliably propagated events during a train of action potentials in dopaminergic neurons. We reasoned that such stochastic propagation of action potentials could result from the availability of sodium and potassium channels at any given time during a barrage of sEPSPs, and so be controlled by the time and voltage dependence of channel activation and inactivation processes. To test the involvement of $I_{\mathrm{A} \text {-like }}$ potassium channels in this behavior, we found that the pharmacological blockade of $I_{\mathrm{A} \text {-like }}$ channels prevented the failure of action potential backpropagation, indicating that the availability of $I_{\mathrm{A}-\text { like }}$ channels contributes to the balance of trans-membrane currents that shape AP backpropagation. In support of this notion, we directly demonstrated the activation of somatic $I_{\mathrm{A}-\text {-like }}$ channels by action potential-shaped waveforms. Interestingly, $I_{\mathrm{A}-\text { like }}$ channels were found to be nonuniformly distributed, with a high somatic density of channels, a subcellular distribution in stark contrast to the polarized dendritic distribution found in cortical pyramidal neurons (Hoffman et al., 1997; Misonou et al., 2004). Furthermore, we find that the inactivation properties of $I_{\mathrm{A}-\text { like }}$ channels are determined in a regionspecific manner, allowing a significantly greater fraction of somatic $I_{\mathrm{A}-\text { like }}$ channels to be available for activation across the voltage range over which action potentials are generated. Thus, it appears that somatic $I_{\mathrm{A} \text {-like }}$ channels are ideally situated to gate action potentials as they spread from axonal site of origin toward the soma and, therefore, provide a mechanism by which small deviations of the membrane potential could influence the backpropagation of action potentials from the axon-bearing dendrite of dopaminergic neurons.

The application of dopamine increased the proportion of APs that failed to invade somatic and nonaxon-bearing dendritic sites. We found that the membrane potential hyperpolarization mediated by activation of $\mathrm{D}_{2}$ receptors was both necessary and sufficient to explain this behavior. We therefore, propose that dopamine gates action potential backpropagation by influencing the voltage-dependent balance of somatodendritic ion channel availability. In support of this notion, we observed that blockade of $I_{\mathrm{A} \text {-like }}$ channels prevented the dopamine-mediated increase of AP failure. We suggest that the potassium channels involved in dopaminegating of action potential backpropagation are predominantly somatic $I_{\mathrm{A} \text {-like }}$ channels, because the voltage-dependent inactivation properties of somatic, but not dendritic $I_{\mathrm{A}-\text { like }}$ channels provide a larger fraction of available channels at firing threshold.

Dopamine, released from somatodendritic sites has been suggested to inhibit the firing rate of dopaminergic neurons (Aghajanian and Bunney, 1977; Ruffieux and Schultz, 1980; Pucak and Grace, 1994; Tepper et al., 1997; Paladini et al., 2003; Beckstead et al., 2004). In addition, previous indirect evidence suggests that dopamine may influence the spread of action potentials from the axon to the soma of dopaminergic neurons in vivo, because extracellular recordings have revealed that the fraction of anti- dromic action potentials with a waveform characteristic of isolated axonal spikes is increased by activation of and decreased by compromising the dopaminergic system (Trent and Tepper, 1991; Tepper et al., 1997). Our data provide a mechanistic framework for the interpretation of these findings and reveal that the failure of AP backpropagation occurs in response to physiological patterns of input and not solely in response to antidromic stimuli (Trent and Tepper, 1991; Häusser et al., 1995; Tepper et al., 1997). Moreover, when action potential firing is evoked by sEPSPs in vitro, action potential waveforms are directly comparable with those observed in vivo, suggesting that action potential backpropagation in dopaminergic neurons is likely to be temporally imprecise and labile in vivo. Because the nonaxonal vesicular release of dopamine from somatodendritic sites occurs in an action potential- and calcium-dependent manner (Beckstead et al., 2004), we suggest that the spread of action potentials from the axon-bearing dendrite to the soma and throughout the dendritic tree may control dopamine release in vivo. We therefore propose that dopamine released from somatodendritic stores could act to gate action potential backpropagation by enhancing the failure of propagation. Under such conditions, dopamine-mediated autoinhibition would be self-regulated, because failed APs do not evoke calcium-dependent processes, and so will be incapable of triggering further calcium-dependent somatodendritic dopamine release. We suggest that such a mechanism will not only control somatodendritic autoinhibition, and therefore play a role in the control of dopamine release at nigrostriatal axon terminals, but also function to control the local levels of dopamine within the substantia nigra. The local regulation of SNR dopamine will act directly to control the GABAergic output neurons of the basal 
ganglia (Ruffieux and Schultz, 1980; Waszcak and Walters, 1983, 1986).

\section{References}

Aghajanian GK, Bunney BS (1977) Dopamine "autoreceptors": pharmacological characterization by microiontophoretic single cell recording studies. Naunyn Schmiedebergs Arch Pharmacol 297:1-7.

Beckstead MJ, Grandy DK, Wickman K, Williams JT (2004) Vesicular dopamine release elicits an inhibitory postsynaptic current in midbrain dopamine neurons. Neuron 42:939-946.

Bernard C, Johnston D (2003) Distance-dependent modifiable threshold for action potential back-propagation in hippocampal dendrites. J Neurophysiol 90:1807-1816.

Bernard C, Anderson A, Becker A, Poolos NP, Beck H, Johnston D (2004) Acquired dendritic channelopathy in temporal lobe epilepsy. Science 305:532-535.

Bjorklund A, Lindvall O (1975) Dopamine in dendrites of substantia nigra neurons: suggestions for a role in dendritic terminals. Brain Res 83:531-537.

Carr DB, Day M, Cantrell AR, Held J, Scheuer T, Catterall WA, Surmeier DJ (2003) Transmitter modulation of slow, activity-dependent alterations in sodium channel availability endows neurons with a novel form of cellular plasticity. Neuron 39:793-806.

Cheramy A, Leviel V, Glowinski J (1981) Dendritic release of dopamine in the substantia nigra. Nature 289:537-542.

Falkenburger BH, Barstow KL, Mintz IM (2001) Dendrodendritic inhibition through reversal of dopamine transport. Science 293:2465-2470.

Geffen LB, Jessell TM, Cuello AC, Iversen LL (1976) Release of dopamine from dendrites in rat substantia nigra. Nature 260:258-260.

Grace AA (1990) Evidence for the functional compartmentalization of spike generating regions of rat midbrain dopamine neurons recorded in vitro. Brain Res 524:31-41.

Grace AA, Bunney BS (1984a) The control of firing pattern in nigral dopamine neurons: single spike firing. J Neurosci 4:2866-2876.

Grace AA, Bunney BS (1984b) The control of firing pattern in nigral dopamine neurons: burst firing. J Neurosci 4:2877-2890.

Guyenet PG, Aghajanian GK (1978) Antidromic identification of dopaminergic and other output neurons of the rat substantia nigra. Brain Res 150:69-84.

Häusser M, Stuart G, Racca C, Sakmann B (1995) Axonal initiation and active dendritic propagation of action potentials in substantia nigra neurons. Neuron 15:637-647.

Häusser M, Spruston N, Stuart GJ (2000) Diversity and dynamics of dendritic signaling. Science 290:739-744.

Hoffman DA, Magee JC, Colbert CM, Johnston D (1997) $\mathrm{K}^{+}$channel regulation of signal propagation in dendrites of hippocampal pyramidal neurons. Nature 387:869-875

Iribe Y, Moore K, Pang KCH, Tepper JM (1999) Subthalamic stimulationinduced synaptic responses in substantia nigra pars compacta dopaminergic neurons in vitro. J Neurophysiol 82:925-933.

Isaacson JS, Walmsley B (1995) Counting quanta: direct measurements of transmitter release at a central synapse. Neuron 15:875-884.

Johnston D, Hoffman DA, Magee JC, Poolos NP, Watanabe S, Colbert CM, Migliore M (2000) Dendritic potassium channels in hippocampal pyramidal neurons. J Physiol (Lond) 525:75-81.

Juraska JM, Wilson CJ, Groves PM (1977) The substantia nigra of the rat: a Golgi study. J Comp Neurol 172:585-600.

Lacey MG, Mercuri NB, North RA (1987) Dopamine acts on D2 receptors to increase potassium conductance in neurones of the rat substantia nigra zona compacta. J Physiol (Lond) 392:397-416.

Lacey MG, Mercuri NB, North RA (1989) Two cell types in rat substantia nigra zona compacta distinguished by membrane properties and the actions of dopamine and opioids. J Neurosci 9:1233-1241.

Liss B, Franz O, Sewing S, Bruns R, Neuhoff H, Roeper J (2001) Tuning pacemaker frequency of individual dopaminergic neurons by Kv4.3L and KChip3.1 transcription. EMBO J 20:5715-57124.

Magee JC, Johnston D (1997) A synaptically controlled, associative signal for Hebbian plasticity in hippocampal neurons. Science 275:209-213.

Misonou H, Mohapatra DP, Park EW, Leung V, Zhen D, Misonou K, Anderson AE, Trimmer JS (2004) Regulation of ion channel localization and phosphorylation by neuronal activity. Nat Neurosci 7:711-718.

Nakanishi H, Kita H, Kitai ST (1987) Intracellular study of rat substantia nigra pars reticulata neurons in an in vitro slice preparation: electrical membrane properties and response characteristics to subthalamic stimulation. Brain Res 437:45-55.

Paladini CA, Robinson S, Morikawa H, Williams JT, Palmiter RD (2003) Dopamine controls the firing pattern of dopamine neurons via a network feedback mechanism. Proc Natl Acad Sci USA 100:2866-2871.

Palmer LM, Stuart GJ (2006) Site of action potential initiation in layer 5 pyramidal neurons. J Neurosci 26:1854-1863.

Pucak ML, Grace AA (1994) Evidence that systemically administered dopamine antagonists activate dopamine neuron firing primarily by blockade of somatodendritic autoreceptors. J Pharmacol Exp Ther 271:1181-1192.

Richards CD, Shiroyama T, Kitai ST (1997) Electrophysiological and immunocytochemical characterization of GABA and dopamine neurons in the substantia nigra of the rat. Neuroscience 80:545-557.

Ruffieux A, Schultz W (1980) Dopaminergic activation of reticulata neurones in the substantia nigra. Nature 285:240-241.

Schultz W (2006) Behavioral theories and the neurophysiology of reward. Annu Rev Psychol 57:87-115.

Shu Y, Hasenstaub A, Duque A, Yu Y, McCormick DA (2006) Modulation of intracortical synaptic potentials by presynaptic somatic membrane potential. Nature 441:761-765.

Stuart G, Sakmann B (1995) Amplification of EPSPs by axosomatic sodium channels in neocortical pyramidal neurons. Neuron 15:1065-1076.

Stuart G, Spruston N, Sakmann B, Häusser M (1997) Action potential initiation and backpropagation in neurons of the mammalian CNS. Trends Neurosci 20:125-131.

Stuart GJ, Häusser M (2001) Dendritic coincidence detection of EPSPs and action potentials. Nat Neurosci 4:63-71.

Stuart GJ, Dodt HU, Sakmann B (1993) Patch-clamp recordings from the soma and dendrites of neurons in brain slices using infrared video microscopy. Pflügers Arch 423:511-518.

Tepper JM, Sawyer SF, Groves PM (1987) Electrophysiologically identified nigral dopaminergic neurons intracellularly labeled with HRP: lightmicroscopic analysis. J Neurosci 7:2794-2806.

Tepper JM, Sun BC, Martin LP, Creese I (1997) Functional roles of Dopamine D2 and D3 autoreceptors on nigrostriatal neurons analyzed by antisense knockdown in vivo. J Neurosci 17:2519-2530.

Tobler PN, Fiorillo CD, Schultz W (2005) Adaptive coding of reward value by dopamine neurons. Science 307:1642-1645.

Trent F, Tepper JM (1991) Dorsal raphe stimulation modifies striatalevoked antidromic invasion of nigral dopaminergic neurons in vivo. Exp Brain Res 84:620-630.

Vetter P, Roth A, Hausser M (2001) Propagation of action potentials in dendrites depends on dendritic morphology. J Neurophysiol 85:926-937.

Waszcak BL, Walters JR (1983) Dopamine modulation of the effects of gamma-aminobutyric acid on substantia nigra pars reticulata neurons. Science 220:218-221.

Waszczak BL, Walters JR (1986) Endogenous dopamine can modulate inhibition of substantia nigra pars reticulata neurons elicited by GABA iontophoresis or striatal stimulation. J Neurosci 6:120-126.

Wichmann T, DeLong MR (1996) Functional and pathophysiological models of the basal ganglia. Curr Opin Neurobiol 6:751-758.

Wichmann T, Bergman H, DeLong MR (1994) The primate subthalamic nucleus. I. Functional properties in intact animals. J Neurophysiol 72:494-506.

Williams SR, Stuart GJ (2000a) Action potential backpropagation and somatodendritic distribution of ion channels in thalamocortical neurons. J Neurosci 20:1307-1317.

Williams SR, Stuart GJ (2000b) Backpropagation of physiological spike trains in neocortical pyramidal neurons: implications for temporal coding in dendrites. J Neurosci 20:8238-8246.

Williams SR, Stuart GJ (2002) Dependence of EPSP efficacy on synapse location in neocortical pyramidal neurons. Science 295:1907-1910.

Williams SR, Buhl EH, Mody I (1998) The dynamics of synchronized neurotransmitter release determined from compound spontaneous IPSCs in rat dentate granule neurones in vitro. J Physiol (Lond) 510:477-497.

Yang F, Feng L, Zheng F, Johnson SW, Du J, Shen L, Wu CP, Lu B (2001) GDNF acutely modulates excitability and A-type $\mathrm{K}(+)$ channels in midbrain dopaminergic neurons. Nat Neurosci 4:1071-1078.

Yung WH, Hausser MA, Jack JJ (1991) Electrophysiology of dopaminergic and non-dopaminergic neurones of the guinea-pig substantia nigra pars compacta in vitro. J Physiol (Lond) 436:643-667. 